\title{
DIAGNÓSTICOS DE ENFERMAGEM DA NANDA-I COM BASE NOS PROBLEMAS SEGUNDO TEORIA DE WANDA HORTA*
}

\author{
Isabela Ubaldo', Eliane Matos², Nádia Chiodelli Salum³
}

${ }^{1}$ Enfermeira. Mestre Profissional em Gestão do Cuidado em Enfermagem. Hospital Universitário da Universidade Federal de Santa Catarina. Florianópolis, SC, Brasil.

${ }^{2}$ Enfermeira. Doutora em Enfermagem. Docente do Programa de Pós-Graduação Gestão do Cuidado em Enfermagem. Universidade Federal de Santa Catarina. Florianópolis, SC, Brasil.

${ }^{3}$ Enfermeira. Doutora em Enfermagem. Docente do Programa de Pós-Graduação Gestão do Cuidado em Enfermagem. Universidade Federal de Santa Catarina. Florianópolis, SC, Brasil.

RESUMO: O objetivo deste estudo foi levantar os problemas de enfermagem, frequentemente identificados nos históricos de enfermagem dos pacientes internados em unidades clínicas, relacionando-os com a classificação diagnóstica North American Nursing Diagnosis Association-International. Trata-se de uma pesquisa descritiva de abordagem quantitativa. Utilizou-se a análise documental para coleta de dados. Os dados foram coletados no mês de agosto de 2011, nos prontuários de 134 pacientes nos quais foram identificados 141 problemas de enfermagem, sendo a partir destes identificados 35 diagnósticos diferentes. Estes se aproximam majoritariamente de diagnósticos relacionados às necessidades psicobiológicas (117) e psicossociais (24). Não foram identificados diagnósticos relacionados às necessidades psicoespirituais. Conclui-se que enfermeiros priorizam os problemas físicos dos pacientes sob seus cuidados, muitas vezes em detrimento dos problemas sociais e espirituais.

DESCRITORES: Clínica médica; Enfermagem; Diagnóstico de enfermagem.

\section{NANDA-I NURSING DIAGNOSES BASED ON WANDA HORTA'S THEORY}

ABSTRACT: The present study aimed to investigate nursing problems usually identified in the nursing records of patients admitted to medical units, relating them to the North American Nursing Diagnosis AssociationInternational (NANDA-I) diagnostic classification. It is a quantitative descriptive study. Documental analysis was used for data collection. Data was collected in August 2011, from the records of 134 patients, and 141 nursing problems were detected, and 35 different diagnoses were identified Most of them concern diagnoses related to psychobiological (117) and psychosocial (24) needs. No diagnoses related to psychospiritual needs were identified. It is concluded that nurses prioritize the physical problems of the patients at the expense of social and spiritual problems.

DESCRIPTORS: Medical practice; Nursing; Nursing diagnosis.

\section{DIAGNÓSTICOS DE ENFERMERÍA DE NANDA-I CON BASE EN LOS PROBLEMAS SEGÚN TEORÍA DE WANDA HORTA}

RESUMEN: El objetivo del estudio fue investigar los problemas de enfermería, frecuentemente identificados en los históricos de enfermería de pacientes internados en unidades clínicas, relacionándolos con la clasificación diagnóstica - North American Nursing Diagnosis Association - International. Es una investigación descriptiva de abordaje cuantitativo. Fue utilizado el análisis documental para obtener los datos, que fueron recogidos en el mes de agosto de 2011, en los prontuarios de 134 pacientes. De ellos, 141 fueron identificados como asociados a problemas de enfermería, totalizándose 35 diagnósticos distintos. Estos se acercan majoritariamente de diagnósticos referentes a las necesidades psicobiológicas (117) y psicosociales (24). No fueron identificados diagnósticos referentes a las necesidades psicoespirituales. Se concluye que enfermeros priorizan los problemas físicos de los pacientes bajo sus cuidados, muchas veces en detrimento de los problemas sociales y espirituales.

DESCRIPTORES: Clínica médica; Enfermería; Diagnóstico de enfermería.

*Artigo extraído da dissertação de Mestrado intitulada: “O diagnóstico de enfermagem da NANDA Internacional na sistematização da assistência de enfermagem, na clínica médica de um hospital universitário". Universidade Federal de Santa Catarina, 2013.

Autor Correspondente:

Eliane Matos

Universidade Federal de Santa Catarina

R. Professor Ayrton Roberto de Oliveira, 20 ap 302A - 88034-050 - Florianópolis, SC, Brasil

E-mail: elianematos@hotmail.com
Recebido: 24/03/2015

Finalizado: 21/09/2015 


\section{INTRODUÇÃO}

As unidades de clínica médica, presentes na maioria dos hospitais gerais, são importantes ambientes de internação para diagnóstico e tratamento depessoas adultas, em geral portadoras de patologias crônicas e agudas não cirúrgicas. Concentram uma diversidade de pacientes com as mais variadas doenças, alguns deles com alta dependência de cuidados de enfermagem. O aumento da prevalência das doenças crônicas e da longevidade da população brasileira resulta em maior número de internações de pessoas idosas, que trazem consigo uma dependência natural consequente ao envelhecimento ${ }^{(1)}$.

Para responder a essa realidade, os enfermeiros que atuam em clínica médica necessitam de conhecimentos ampliados e capacidade de prever cuidados sistematizados aos pacientes, tanto no sentido de atender às necessidades individuais da clientela, como para desenvolver conhecimentos próprios da profissão(2).

A Sistematização da Assistência de Enfermagem (SAE) e a aplicação do Processo de Enfermagem $(\mathrm{PE})$ vem responder à necessidade de organização dos serviços de enfermagem e do planejamento da assistência baseada na cientificidade para identificar as situações de saúde-doença dos indivíduos e subsidiar as ações de assistência, contribuindo para a promoção, prevenção, recuperação e reabilitação da saúde ${ }^{(3)}$.

Wanda de Aguiar Horta, na década de 1970, foi a pioneira desta reflexão no Brasil, mas foi na segunda metade da década de 90 que as experiências de aplicação da SAE em nossa realidade ganharam força e começaram a se multiplicar atingindo o seu ápice nos anos 2000, sinalizando para o avanço científico da prática de enfermagem no país. A autora desenvolveu seus estudos a partir da teoria da motivação humana de Maslow, que se fundamenta nas necessidades humanas básicas e utiliza a denominação adotada por João Mohana, ou seja, necessidades psicobiológicas, psicossociais e psicoespirituais ${ }^{(2)}$.

As necessidades psicobiológicas concentram: oxigenação; hidratação; eliminação; sono e repouso; nutrição; exercício e atividades físicas; abrigo; mecânica corporal; motilidade; sexualidade, cuidado corporal; integridade cutâneo-mucosa e física; regulação térmica, hormonal, neurológica, hidroeletrolítica, imunológica, crescimento celular, vascular; percepção dos órgãos do sentido; ambiente; terapêutica e locomoção. As necessidades psicossociais: segurança, amor, liberdade, comunicação, criatividade, aprendizagem, gregária, recreação, lazer, espaço, orientação no tempo e espaço, aceitação, autorrealização, autoestima, participação, autoimagem e atenção. As necessidades psicoespirituais: religiosa ou teológica, ética e de filosofia de vida(2).

No ano de 2002, o Conselho Federal da Enfermagem (COFEN), pela Resolução COFEN $358 / 2009$, torna a SAE obrigatória em todas as instituições de saúde em que atua pessoal de enfermagem ${ }^{(3)}$.

Para realizar a $\mathrm{SAE}$, é necessário um suporte teórico que oriente as etapas desse processo, e a Teoria das Necessidades Humanas Básicas (NHB) de Wanda Horta vem sendo a mais utilizada pelas instituições de saúde do país, sendo este referencial adequado pelos enfermeiros a cada realidade $^{(2)}$.

O Processo de Enfermagem (PE) é definido como "a dinâmica das ações sistematizadas e inter-relacionadas, visando à assistência ao ser humano"(4:35). O modelo conta com seis fases distintas: histórico, diagnóstico, plano assistência, plano de cuidado ou prescrição, prognóstico e evolução de enfermagem.

$\mathrm{Na}$ implementação do modelo de Horta, o enfermeiro realiza o histórico de enfermagem nas primeiras horas de internação; destaca os problemas de enfermagem, que de acordo com Horta são "situações ou condições decorrentes dos desequilíbrios das necessidades básicas do indivíduo, família e/ou comunidade, que exigem do enfermeiro assistência profissional" (4:39).

Com base nos problemas, o enfermeiro elabora a prescrição de cuidados, que é implementada pela equipe de enfermagem oferecendo a assistência de que o paciente necessita. Na evolução de enfermagem, passo subsequente deste processo, o enfermeiro avalia os resultados dos cuidados de enfermagem, incluindo, excluindo ou modificando as intervenções, de acordo com as respostas do paciente ao cuidado prestado. Neste processo que se atualiza a cada 24 horas, o enfermeiro avalia se os problemas foram ou não resolvidos, se novos problemas surgiram ou se modificaram com o cuidado de enfermagem ${ }^{(5)}$.

O modo como SAE é realizada na instituição, foco deste estudo, com a realização do histórico de enfermagem - definição de problemas do paciente - prescrição - implementação dos cuidados evolução, em um processo contínuo, possibilita a previsão de cuidados individualizados, porém 
não garante o cumprimento da Resolução COFEN 358/2009 ${ }^{(3)}$, que estabelece como obrigatória na SAE. Já a fase diagnóstica na instituição em estudo é representada pela fase de definição de problemas.

O diagnóstico de enfermagem (DE) é considerado a etapa mais complexa do processo de enfermagem, constituindo-se em importante desafio para o enfermeiro por requerer dele o pensamento crítico e conhecimentos técnicocientíficos para interpretação dos dados obtidos no exame físico e nas informações fornecidas pelo paciente durante a entrevista. A formulação adequada do DE direciona o planejamento e implementação dos cuidados, assim como possibilita analisar e interpretar criteriosamente a evolução do paciente neste processo ${ }^{(6)}$.

Existem na realidade algumas classificações diagnósticas disponíveis ${ }^{(7)}$. A North American Nursing Diagnosis Association-International (NANDA-I) é uma das classificações mais conhecidas na realidade brasileira ${ }^{(8)}$.

Entendendo a necessidade de avançar na adoção da etapa diagnóstica na SAE e com a perspectiva de iniciar a aplicação desta etapa na instituição em estudo, buscou-se levantar os problemas de enfermagem, frequentemente identificados pelo enfermeiro nos históricos de enfermagem dos pacientes internados em unidades de clínica médica, relacionando-os à classificação diagnóstica da NANDA-I.

\section{MÉTODO}

Pesquisa descritiva de abordagem quantitativa, transversal, que buscou levantar os problemas de enfermagem, frequentemente identificados pelo enfermeiro nos históricos de enfermagem dos pacientes internados em unidades de clínica médica, relacionando-os à classificação diagnóstica da NANDA-I.

O campo de pesquisa foram três unidades de clínica médica de um hospital geral universitário, de médio porte, localizado em uma capital de estado do sul do Brasil, as quais totalizam 77 leitos distribuídos nas especialidades de cardiologia, neurologia, reumatologia, pneumologia, nefrologia, gastrenterologia, endocrinologia, hematologia e clínica médica propriamente dita. Nestas unidades atuam 24 enfermeiros, os quais realizam diariamente a $\mathrm{SAE}$, utilizandose do referencial das Necessidades Humanas Básicas, aplicando o histórico de enfermagem na internação do paciente, momento em que também definem os principais problemas de enfermagem e estabelecem o plano de cuidados/prescrição de enfermagem. A partir daí, em um processo diário e contínuo, realizam a evolução do paciente com base nos cuidados prestados, e, se necessário, atualizam a prescrição de enfermagem.

O hospital em estudo, de uma instituição federal de ensino, possui 319 leitos nas áreas de cirurgia, clínica médica, maternidade, pediatria e neonatologia, ginecologia e tratamento intensivo, e atende exclusivamente pelo Sistema Único de Saúde. A opção por investigar o fenômeno nas unidades de clínica médica decorreu da inserção de uma das pesquisadoras em uma das três unidades existentes na instituição.

A coleta de dados aconteceu nos meses de agosto e setembro de 2011, em dois períodos de uma semana com intervalo de 15 dias entre elas, objetivando com isso um número maior de registros e de casos analisados pela rotatividade de pacientes. Foram convidados a participar do estudo todos os pacientes internados no período de coleta de dados, independente do motivo de internação, sendo que não houve recusa à participação. Os pacientes ou seus representantes legais foram esclarecidos quanto aos objetivos e métodos da pesquisa, e autorizaram a consulta nos prontuários por meio do Termo de Consentimento Livre e Esclarecido.

Utilizou-se como critério de exclusão a inexistência de histórico de enfermagem no momento da pesquisa, ou seja, nas situações em que o paciente estava internado há menos de 24 horas. Foram excluídos ainda os prontuários de pacientes que haviam sido analisados na primeira semana de coleta de dados, e que permaneciam internados no segundo momento de coleta de dados. Fizeram parte do estudo 134 prontuários de pacientes internados.

A coleta de dados foi realizada por meio da leitura do Histórico de Enfermagem, quando foram compilados todos os problemas de enfermagem identificados pelos enfermeiros na entrevista e exame físico.

Para análise dos dados, os problemas identificados no histórico do paciente foram agrupados e registrados em uma planilha do software Exce/®, organizadas em Necessidades Psicobiológicas, Psicossociais e Psicoespirituais. Os agrupamentos de problemas foram comparados à nomenclatura de diagnósticos de enfermagem da NANDA-I, sendo novamente organizados como diagnósticos de enfermagem. 
Foram descritas as frequências relativas e absolutas dos achados que foram analisados segundo o referencial teórico.

O estudo foi aprovado pelo Comitê de Ética em Pesquisas com Seres Humanos (CEPSH) da UFSC, com parecer $n^{\circ} 2134 / 2011$, seguindo todas as determinações da Resolução 196/1996(9) e suas complementares, vigentes à época da pesquisa.

\section{RESULTADOS}

Quanto à caracterização dos pacientes internados nas unidades de clínica médica, constata-se que $80(59,7 \%)$ pertenciam ao sexo feminino e 54 (40,3\%), ao sexo masculino. A idade variou entre 16 e 98 anos, sendo que 44\% dos pacientes possuíam idade acima de 60 anos.

Nos 134 históricos de enfermagem analisados, foram identificados 141 diferentes problemas de enfermagem. Dentre estes, 117 (82,98\%) concentravam-se no campo das necessidades psicobiológicas; 24 (17,02\%) diziam respeito às necessidades psicossociais. Não foram identificados problemas relacionados às necessidades psicoespirituais, conforme mostra o Quadro 1.

Quadro 1 - Problemas relacionados às necessidades psicobiológicas/psicossociais identificadas nos históricos de Enfermagem e relação com diagnósticos de enfermagem (NANDA-I). Florianópolis, SC, Brasil, 2012

\begin{tabular}{|c|c|c|c|}
\hline Necessidades Psicobiológicas $(n=117)$ & $\mathbf{N}$ & $\%$ & Diagnóstico relacionado \\
\hline $\begin{array}{l}\text { Regulação } \\
\text { Níveis pressóricos aumentados (vascular) } \\
\text { Níveis glicêmicos aumentados (hormonal) } \\
\text { Presença de edemas (eletrolítica) }\end{array}$ & 21 & 14,9 & $\begin{array}{l}\text { Risco de débito cardíaco diminuído; Intolerância } \\
\text { à atividade, Perfusão tissular ineficaz, Risco de } \\
\text { glicemia instável. }\end{array}$ \\
\hline $\begin{array}{l}\text { Oxigenação } \\
\text { Queixa de dispneia } \\
\text { Uso de suplemento de oxigênio por } \\
\text { cateter nasal }\end{array}$ & 16 & 11,34 & $\begin{array}{l}\text { Troca de gases prejudicada, Padrão respiratório } \\
\text { ineficaz, Intolerância à atividade, Risco de } \\
\text { intolerância à atividade. }\end{array}$ \\
\hline $\begin{array}{l}\text { Integridade cutaneomucosa } \\
\text { Úlceras por pressão } \\
\text { Problemas relacionados à locomoção }\end{array}$ & 14 & 9,93 & $\begin{array}{l}\text { Risco de integridade da pele prejudicada, } \\
\text { Integridade da pele prejudicada, Integridade } \\
\text { tissular prejudicada, Risco de infecção; } \\
\text { Mobilidade física prejudicada, Mobilidade no } \\
\text { leito prejudicada. }\end{array}$ \\
\hline $\begin{array}{l}\text { Terapêutica } \\
\text { Manutenção de acesso venoso }\end{array}$ & 14 & 9,93 & Risco de Infecção. \\
\hline $\begin{array}{l}\text { Percepção dos órgãos do sentido } \\
\text { Queixas álgicas }\end{array}$ & 13 & 9,22 & Dor aguda, Dor crônica. \\
\hline $\begin{array}{l}\text { Eliminação } \\
\text { Disfunção de esfíncteres }\end{array}$ & 11 & 7,8 & $\begin{array}{l}\text { Constipação, risco de constipação, Motilidade } \\
\text { gastrintestinal disfuncional, } \\
\text { Incontinência fecal, Incontinência urinária } \\
\text { funcional, Retenção urinária, Eliminação urinária } \\
\text { prejudicada, Risco de infecção. }\end{array}$ \\
\hline $\begin{array}{l}\text { Ambiente } \\
\text { Risco de queda } \\
\text { Cuidado corporal: necessidade de auxílio } \\
\text { da equipe de enfermagem para banho }\end{array}$ & 10 & 7,1 & $\begin{array}{l}\text { Risco de quedas. } \\
\text { Déficit no autocuidado para banho. }\end{array}$ \\
\hline $\begin{array}{l}\text { Nutrição } \\
\text { Queixa de inapetência }\end{array}$ & 10 & 7,1 & $\begin{array}{l}\text { Nutrição desequilibrada: menos que as } \\
\text { necessidades corporais. }\end{array}$ \\
\hline $\begin{array}{l}\text { Hidratação } \\
\text { Baixa ingesta hídrica durante a } \\
\text { hospitalização }\end{array}$ & 5 & 3,53 & $\begin{array}{l}\text { Volume de líquidos deficiente, Risco de volume } \\
\text { de líquidos deficiente. }\end{array}$ \\
\hline $\begin{array}{l}\text { Sono e repouso } \\
\text { Dificuldade para conciliar o sono }\end{array}$ & 3 & 2,13 & $\begin{array}{l}\text { Conforto prejudicado, Insônia, Padrão de sono } \\
\text { prejudicado. }\end{array}$ \\
\hline Necessidades Psicossociais $(n=24)$ & $\mathbf{N}$ & $\%$ & \\
\hline Segurança & 24 & 17,02 & Medo; Enfrentamento Ineficaz, Ansiedade. \\
\hline \multirow[t]{2}{*}{ Necessidade psicoespirituais } & $\mathbf{N}$ & $\%$ & \\
\hline & $\mathbf{0}$ & $\mathbf{0}$ & \\
\hline Total & 141 & 100 & \\
\hline
\end{tabular}


As necessidades psicossociais foram apresentadas basicamente naquelas situações relacionadas à permanência do paciente longe do seu contexto, de seus familiares e de suas atividades cotidianas pela internação, expressas em labilidade emocional, ansiedade, choro, isolamento, medo, angústia, baixa estima, agressividade, depressão, tristeza e saudade da família. Os problemas relacionados às necessidades espirituais não foram identificados no estudo.

\section{DISCUSSÃO}

Observa-se na análise a predominância de pacientes com idade inferior a 60 anos, internados nas unidades de clínica médica, situação contrária à experiência prática em que há predominância de pessoas idosas nestas unidades. Há que se considerar o elevado número de pacientes jovens acometidos por problemas imunológicos internados no momento da pesquisa. Conclusões acerca do perfil dos pacientes internados em clínica médica necessitam de novas investigações que permitam avaliar sazonalidade e possíveis mudanças no perfil dos pacientes.

Predominam na avaliação dos enfermeiros os problemas relacionados às necessidades psicobiológicas, mesmo que o modelo teórico de Horta reconheça o ser humano como portador de necessidades biopsicoespirituais. Esse dado reforça a formação dos profissionais de enfermagem, ainda hoje centrada no conhecimento da biomedicina e no cuidado de enfermagem voltado para o corpo do paciente, e salientam a importância da profissão investir em modelos de atenção integral que considerem as múltiplas dimensões do ser humano(10-11).

Dentre os problemas de enfermagem, constata-se no grupo de pacientes estudados a predominância daqueles relacionadosà regulação, oxigenação e integridade cutaneomucosa. Dentre as necessidades de regulação, destaca-se o aumento dos níveis pressóricos e glicêmicos e a presença de edema segmentos corporais, compatíveis com o crescimento de doenças crônicas em nossa realidade ${ }^{(12)}$.

Outros destaques estão relacionados aos problemas de oxigenação, em pacientes internados na instituição estudada, em função da referência no atendimento aos pneumopatas. Também, a prevenção/cuidado com as úlceras por pressão, que acometem com frequência pacientes confinados no leito por um período longo e/ou em pacientes com dificuldades sensoriais, motoras ou cognitivas prejudicadas podem aumentar a chance de complicações. Neste sentido, compete à equipe de enfermagem buscar efetivar cuidados individuais, centralizados nas necessidades essenciais de cada paciente, a se iniciar na admissão, independentemente da origem de cada idoso ${ }^{(13)}$.

Estudo semelhante identificou estes DE em 196 prontuários de pacientes idosos; sendo que o Risco de integridade da pele prejudicada foi o diagnóstico de maior expressão, afetando 46,4\% da população do estudo seguidos de Integridade da pele prejudicada e integridade tissular prejudicada $^{(14)}$.

Administração de medicamentos invasivos e manutenção de acesso venoso estão entre os problemas de enfermagem listados por enfermeiros, em decorrência do risco de infecção pela solução de continuidade. Corroborando com dados deste estudo, o Risco de infecção foi prevalente $(58,3 \%)$ em pesquisa que teve por objetivo identificar os DE em uma unidade hospitalar de atendimento médico cirúrgico(15). O diagnóstico de risco de infecção pode também estar relacionado ao diagnóstico de integridade cutâneo mucosa ${ }^{(14)}$.

Destacou-se ainda a percepção dolorosa em diversos segmentos do corpo representado pelo Domínio Conforto da Taxonomia II da NANDA-I, dado que confirma achados de estudo anterior que apontou a dor como o segundo diagnóstico na ordem de prevalência em unidade de clínica médica ${ }^{(16)}$. A dor constitui-se em importante sinal vital, que necessita atenção profissional, uma vez que sua prevenção e controle têm relação direta com a qualidade da assistência ${ }^{(17)}$.

Outros problemas de grande importância também foram descritos pelos enfermeiros, porém com menor frequência, tais como os relacionados às eliminações (intestinais $\mathrm{e}$ vesicais); distúrbios nutricionais, relacionados à ingesta hidro alimentar e aos distúrbios gástricos como náusea, vômito, incapacidade para alimentarem-se sem auxílio, necessidade de sondagens e abstenção da dieta, que no estudo apareceu com uma frequência considerável à tendência dos pacientes a apresentar ingesta hidro alimentar insuficiente. Sobre esse aspecto, é importante considerar que a hospitalização traz consigo mudanças no hábito alimentar que por vezes torna-se um problema de enfermagem. Nestes casos os enfermeiros devem estar atentos para buscar soluções individualizadas para restabelecer a ingesta e reduzir riscos de 
complicações.

A insônia associada ao ambiente hospitalar também foi um achado do estudo. Outras investigações apontam para o mesmo problema ${ }^{(18)}$ e neste sentido é preciso observar que a alteração no padrão do sono em pacientes internados, pode estar relacionada a fatores fisiopatológicos, ao tratamento e a situações pessoais ou ambientais ${ }^{(19)}$.

Outra preocupação presente no estudo diz respeito à segurança e ao risco para quedas associada ao grau de dependência dos pacientes. Essa condição dos pacientes tem chamado a atenção dos enfermeiros para a necessidade de vigilância e a adoção de estratégias de prevenção, com registros e monitoramento de casos com impacto na segurança do paciente $\mathrm{p}^{(20-21)}$.

Com relação às Necessidades Psicossociais e Psicoespirituais, embora ainda pouco abordadas pelos enfermeiros, percebe-se a preocupação com o medo e ansiedade presente no adoecimento e na internação hospitalar. Nesta perspectiva, autores destacam a dificuldade dos enfermeiros em lidar com o ser biopsicossocial e espiritual, dispensando na assistência de enfermagem, prioritariamente, cuidados técnicos em detrimento da assistência holística ao paciente ${ }^{(10)}$.

Cabe destacar nesta discussão que os hospitais gerais e as unidades de clínica médica concentram uma maioria de pacientes com problemas de saúde que tem relação estreita com as necessidades psicobiológicas ${ }^{(5)}$. As internações geralmente decorrem de um problema clínico a ser investigado e/ou tratado, o que demanda uma variedade de cuidados de enfermagem relacionados ao corpo físico do paciente. Entretanto, estudos que abordam as $\mathrm{NHB}^{(6,11,12,14,16,21)}$ constatam que o olhar da enfermagem hospitalar está centrado basicamente nesta esfera das necessidades. Assim como os achados desta pesquisa, estes estudos evidenciam que a teorização acerca da interrelação entre as necessidades psicobiológicas, psicossociais e psicoespirituais não tem sido apropriadamente compreendida pelos enfermeiros. A teoria das NHB assume que o não atendimento a uma necessidade ou a um grupo delas, interfere no conjunto das necessidades do ser humano multidimensional(5).

A atenção ao ser humano com carência de cuidados de saúde, o planejamento das intervenções de enfermagem deve perseguir a integralidadedaassistênciae evitaro reducionismo que trata os seres humanos tão somente como seres doentes, esquecendo que estes são dotados de sentimentos, desejos, medos, ansiedades, convicções religiosas e outras que se fazem presentes e são frequentemente afetadas quando se instala uma doença física. A concepção do ser humano integral é uma condição norteadora dos profissionais e instituições que integram o Sistema Único de Saúde.

\section{CONCLUSÕES}

A investigação dos problemas de enfermagem predominantes em pacientes internados em clínica médica e sua relação à classificação diagnóstica da NANDA -I permitiu identificar, pela aproximação destes aos fatores relacionados/ risco e características definidoras, uma série de diagnósticos em pacientes internados em clínica médica.

Os diagnósticos identificados concentram-se na esfera biológica, mostrando o destaque que os enfermeiros dão aos problemas físicos dos pacientes sob seus cuidados, em detrimento, muitas vezes, dos problemas sociais e espirituais. Não desqualificando a necessidade de cuidados com o corpo do paciente, entendemos que é necessário avançar na direção do ser humano multidimensional com necessidades biopsicossociais e espirituais, para se tenha assistência de melhor qualidade.

Relacionando os problemas de enfermagem mencionados pelos enfermeiros nos históricos de enfermagem dos pacientes, com as características definidoras e fatores relacionados da NANDA-I, foram identificados 35 diagnósticos de enfermagem. De acordo com a experiência dos pesquisadores, os diagnósticos apresentados tem aderência à realidade das unidades de clínica médica, dando conta de explicar a necessidade da maioria dos cuidados dispensados aos pacientes internados nesta área assistencial.

Recomenda-se, no entanto, a realização de novos estudos para fortalecer, ampliar ou modificar os achados atuais. Destaca-se também a importância de expandir a pesquisa incluindo a busca de dados na evolução do paciente, realizada diariamente pelos enfermeiros. Isso porque, ao longo da internação, os pacientes acabam modificando sua condição de saúde/doença e/ ou dependência de cuidados de enfermagem, apresentando novos diagnósticos de enfermagem e exigindo novos e diversificados cuidados.

Os achados do estudo possibilitam aos enfermeiros repensar o processo assistencial e a sistematização da assistência de enfermagem com 
vistas a dar maior cientificidade ao seu trabalho, por meio da utilização de uma classificação diagnóstica de enfermagem. A possibilidade de inclusão da etapa diagnóstica à SAE depende, entre outros fatores, de estudos que estimulem os enfermeiros a reorientar a sua prática cotidiana nas instituições de saúde.

\section{REFERÊNCIAS}

1. Both JE, Leite MT, Hildelbrandt LM, Beuter M, Muller LA, Link CL. Qualificação da equipe de enfermagem mediante pesquisa convergente assistencial: contribuições ao cuidado do idoso hospitalizado. Esc Anna Nery. 2014; 18(3):486-95.

2. Cavalcante RB, Otoni A, Bernardes MFVG, Cunha SGS, Santos CS, Silva PC. Experiências de sistematização da assistência de enfermagem no Brasil: um estudo bibliográfico. REUFSM. 2011; 1(3) [acesso em 30 nov 2013]. Disponível: http://cascavel. ufsm.br/revistas/ojs-2.2.2/index.php/reufsm/article/ view/2832/2396.

3. Conselho Federal de Enfermagem. Resolução COFEN-358 de 15 de outubro de 2009. Dispõe sobre a Sistematização da Assistência de Enfermagem e a implementação do Processo de Enfermagem em ambientes, públicos ou privados, em que ocorre o cuidado profissional de Enfermagem. Brasília; 2009 [acesso em 16 jul 2014]. Disponível: http://www.cofen. gov.br/resoluo-cofen-3582009_4384.html.

4. Hospital Universitário. Diretoria de Enfermagem. Modelo assistencial - documentos básicos. [Internet] 1980 [acesso em 26 jun 2015]. Disponível: http://www. hu.ufsc.br/setores/enfermagem/documentos/.

5. Horta WA. Processo de enfermagem. São Paulo: EPU; 1979.

6. Carmo LL, Ramos RS, Oliveira OV, Maciel RO. A identificação de diagnósticos de enfermagem em pacientes de uma unidade de clínica médica: fortalecendo práticas e definindo direções rumo à sistematização da assistência de enfermagem. Revista Hospital Universitário Pedro Ernesto. 2011; 10(Suppl.1) [acesso em 16 jun 2012]. Disponível: http://revista. hupe.uerj.br/detalhe_artigo.asp?id=125.

7. Barros ALBL. Classificações de diagnóstico e intervenção de enfermagem: NANDA-NIC. Acta paul. enferm.[Internet] 2009; 22(spe) [acesso em 23 jun 2013]. Disponível: http://dx.doi.org/10.1590/S010321002009000700003.

8. North American Nursing Diagnosis AssociationInternational. Diagnósticos de enfermagem da NANDA: definições e classificação - 2009-2011. Porto Alegre: Artmed; 2010.
9. Ministério da Saúde (BR). Conselho Nacional de Saúde. Resolução n. 196/96, de 10 outubro 1996. Diretrizes e Normas Regulamentadoras de Pesquisas Envolvendo Seres Humanos. Brasília; 1996 [acesso em 24 jul 2012]. Disponível: http://conselho.saude.gov.br/ resolucoes/1996/Reso196.doc.

10. Prearo C, Gonçalves LS, Vinhando MB, Menezes SL. Percepção do enfermeiro sobre o cuidado prestado aos pacientes portadores de neoplasia. Arq. ciênc. saúde. 2011; 18(1):20-7.

11. Backes MTS, Rosa LM, Fernandes GCM, Becker SG, Meirelles BHS, Santos SMA. Conceitos de saúde e doença ao longo da história sob o olhar epidemiológico e antropológico. Rev. enferm. UERJ. 2009; 1(17):111-7.

12. Silva FM, Budó MLD, Silveira CL, Badke MR, Beuter $M$. Hypertension as a condition of non-disease: the meaning of chronicity in the subjects' perspective. Texto Contexto Enferm. 2013; 22(1):123-31.

13. Freitas MC, Medeiros ABF, Guedes MVC, Almeida PC, Galiza FT, Nogueira JM. Úlcera por pressão em idosos institucionalizados: análise da prevalência e fatores de risco. Rev. Gaúcha Enferm. [Internet]. 2011; 32(1) [acesso em 09 jan 2014]. Disponível: http://dx.doi. org/10.1590/S1983-14472011000100019.

14. Santos ASR, Souza PA, Valle AMD, Cavalcanti ACD, Sá SPC, Santana RF. Caracterização dos diagnósticos de enfermagem identificados em prontuários de idosos: um estudo retrospectivo. Texto Contexto Enferm. 2008; 17(1):141-9.

15. Volpato BM, Cruz DALM. Diagnósticos de enfermagem em pacientes internadas em unidade médico-cirúrgica. Acta paul. enferm. 2007; 20(2):11924.

16. Fontes CMB, Cruz DALM. Diagnósticos de enfermagem documentados para pacientes de clínica médica. Rev. esc. enferm. USP. 2007; 41(3):395-402.

17. Silva VR, Gradin CVC. Avaliação da dor em mulheres com câncer de mama submetidas à exérese da rede linfática axilar. Cogitare Enferm. [Internet] 2010; 15(4) [acesso em 12 fev 2015]. Disponível: http://dx.doi. org/10.5380/ce.v15i4.20360.

18. Carpenito-Moyet, LJ. Manual de diagnósticos de enfermagem. 11 ${ }^{\mathrm{a}}$ ed. Porto Alegre: Artmed; 2008.

19. Avelar AFM, Sales CLS, Bohomol E, Feldman LM, Peterline MAS, Harada MJCS, et al. Dez passos para a segurança do paciente. Conselho Regional de Enfermagem. São Paulo; 2010 [acesso em 16 mai 2012]. Disponível: http://www.coren-sp.gov.br/sites/default/ files/10_passos_seguranca_paciente_0.pdf.

20. Correa AD, Marques IAB, Martinez MC, Laurino OS, Leão ER, Chimentão DMN. Implantação de um 
protocolo para gerenciamento de quedas em hospital: resultados de quatro anos de seguimento. Rev. esc. enferm. USP. 2012; 46(1):67-74.

21. Pinho IC, Siqueira JCBA, Pinho LMO. As percepções do enfermeiro acerca da integralidade da assistência. Rev. Eletr. Enf. [Internet] 2006; 8(1):42-51 [acesso 25 jun. 2015]. Disponível: http://www.fen.ufg.br/revista/ revista8_1/original_05.htm. 